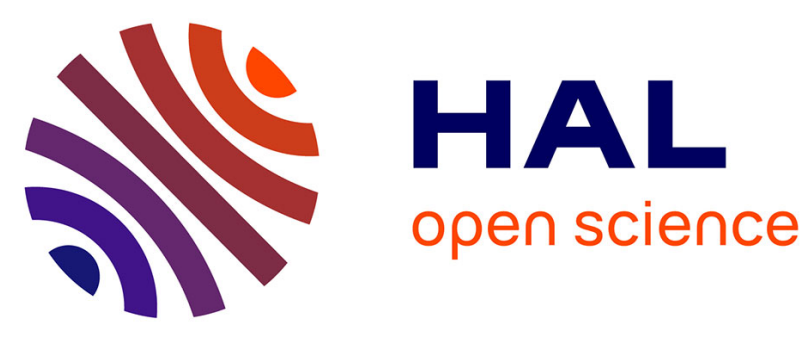

\title{
Evidential network-based multimodal fusion for fall detection
}

\author{
Paulo Armando Cavalcante Aguilar, Jérôme Boudy, Dan Istrate, Hamid \\ Medjahed, Bernadette Dorizzi, João César Moura Mota, Jean-Louis \\ Baldinger, Toufik Guettari, Imad Belfeki
}

\section{To cite this version:}

Paulo Armando Cavalcante Aguilar, Jérôme Boudy, Dan Istrate, Hamid Medjahed, Bernadette Dorizzi, et al.. Evidential network-based multimodal fusion for fall detection. pHealth '11: 8th International Conference on Wearable Micro and Nano Technologies for Personalised Health, Jun 2011, Lyon, France. hal-00707780

\section{HAL Id: hal-00707780 \\ https://hal.science/hal-00707780}

Submitted on 13 Jun 2012

HAL is a multi-disciplinary open access archive for the deposit and dissemination of scientific research documents, whether they are published or not. The documents may come from teaching and research institutions in France or abroad, or from public or private research centers.
L'archive ouverte pluridisciplinaire HAL, est destinée au dépôt et à la diffusion de documents scientifiques de niveau recherche, publiés ou non, émanant des établissements d'enseignement et de recherche français ou étrangers, des laboratoires publics ou privés. 


\title{
Evidential Network-based Multimodal Fusion for Fall Detection
}

\author{
${ }^{1}$ Paulo A. Cavalcante A., ${ }^{1}$ Jerome Boudy, ${ }^{2}$ Dan Istrate, ${ }^{2} \mathrm{H}$. Medjahed, ${ }^{1}$ Bernadette Dorizzi, \\ ${ }^{3}$ J. Cesar M. Mota, ${ }^{1}$ Jean Louis Baldinger, ${ }^{1}$ T. Guettari, ${ }^{1}$ Imad Belfeki. \\ ${ }^{1}$ Electronics and Physics Department, Telecom SudParis, Evry, France \\ ${ }^{2}$ Esigetel, Avon-Fontainebleau, France \\ ${ }^{3}$ Federal University of Ceará, UFC, Fortaleza, Brazil
}

\begin{abstract}
The multi-sensor fusion can provide more accurate and reliable information compared to information from each sensor separately taken. Moreover, the data from multiple heterogeneous sensors present in the medical surveillance systems have different degrees of uncertainty. Among multisensor data fusion techniques, Bayesian methods and evidence theories such as Dempster-Shafer Theory (DST), are commonly used to handle the degree of uncertainty in the fusion processes. Based on a graphic representation of the DST called evidential networks, we propose a structure of heterogeneous multi-sensor fusion for falls detection. The proposed Evidential Network (EN) can handle the uncertainty present in a mobile and a fixed sensor-based remote monitoring systems (fall detection) by fusing them and therefore increasing the fall detection sensitivity compared to the a separated system alone.
\end{abstract}

\section{INTRODUCTION}

It is well known that multi-sensor fusion can provide more accurate and reliable information to detect distress situation for elderly persons living in their home [7]. The potential searched benefits of multi-sensors fusion is the redundancy and complementarity of information. The fusion of redundant information can reduce the overall uncertainty. Moreover, the data from multiple heterogeneous sensors of the medical surveillance systems present varying degrees of uncertainty and confidence $[5,6]$.

Among multi-sensor fusion techniques, we can find Bayesian methods [1] and the Theory of Evidences based on the Dempster-Shafer theory (DST) [2-6, 11-15], which are commonly used to process and estimate degrees of uncertainty in the fusion process [6]. These theories are based on graphical representations: Bayesian Networks [1] and Evidential Networks (EN) [2-6, 15].

The introduction of Bayesian networks for knowledge representation and probabilistic inference has represented a breakthrough in the development of expert systems. However, the limitations of Bayesian networks as a formalism to deal with uncertainty issues consist in the assumption that all data (domain knowledge, accumulated evidence) can be represented by probability functions. In reality, this is not always possible, if the data amount is not sufficient [3].

Indeed the use of Bayesian statistical classifiers in the context of the remote medical monitoring (or "Télévigilance") depends on the availability of adequate databases in order to model with reliability distress situations such as person's falls, cardiac events (e.g. bradycardia, arrhythmia). Modeling a fall's cinematic is not a straightforward task. Indeed the weight, the size and corpulence of the person have a substantial impact on the fall characteristics: in that respect soft falls are particularly difficult to detect. Therefore falls databases are very limited even inexistent due to the lack of records made in real situations.

To this aim, this article investigates and implements an evidential network to detect fall situations and estimate its uncertainty degree through a heterogeneous multi-sensors fusion [5,6]. This network is also appropriate knowing, on one side, the lack of falls databases as here-above stressed, and on the other side, its property allowing direct inference mechanisms on input observations such as actimetric data (body's movement, inclination) and vital data (cardiac frequency and fall index). The Evidential Networks are acyclic-directed graphs similar to Bayesian networks, but they use belief functions instead of probability functions. They are designed to handle uncertainty through the Dempster-Shafer Theory formalism.

In the following sections, first section 2 describes the remote medical monitoring platform constituting our targeted application, then section 3 reminds the basics of DempsterShafer theory, section 4 develops the Evidential Network application to the remote medical monitoring context by explaining how we implement the network and estimate the belief degree of a detected distress event such as hard or soft falls. Finally section 5 provides provisional evaluation results and section 6 concludes on this work with perspectives.

\section{Remote Medical Monitoring Platform}

A remote medical patient's monitoring system with alarm management [7], if integrated in a smart home environment, can use the fusion results of several observation data such as actimetric and vital signals captured by a device worn by the patient, external sensors such as acoustic and presence signals. Such a "Télévigilance" platform exists at Telecom SudParis elaborated with the close collaboration of Esigetel [10] and U558-INSERM (F. Steenkeste [8]). This Alarm management platform is composed of three detection sub-systems or modalities: GARDIEN [8], RFPAT [9] and ANASON [10]. In this new application work on Evidential Network, we first focused on two of these modalities: GARDIEN and RFPAT.

RFPAT system [9, 7] was designed for remote monitoring of vital and actimetric signals recorded on the person. This system is composed of a wearable terminal carried by the patient that can automatically identify distress situations such as falls, abrupt changes of cardiac rhythms (namely bradycardia trend) or person's activity (movements, posture).

The GARDIEN system $[8,7]$ consists in a fixed network of wired or wireless infrared motion sensors placed within the 
smart home environment and external to the person These sensors are activated by body movements which therefore indicate the presence of a person in the area of interest. The person's posture inclination can also be estimated from the combination of two types of infrared sensors, one for horizontal detection field, the other vertical [8].

\section{DemPSTER SHAFER THEORY OF EVIDENCE}

The Dempster Shafer theory (DST) of evidence [2-6, 11-15] was introduced from the work of Dempster and extended by Shafer. It is a generalization of probability theory that allows us to better quantify uncertainty. This theory is based on a number of key propositions which are summarized as follow.

A binary sensor can have either a value of 1 (active) or 0 (inactive). Both values comprise the exhaustive set of mutually exclusive values that the sensor can hold. In DST, this set is called the frame of discernment of the sensor, denoted by $\Theta$. The power set $P(\Theta)$, is the set of all subsets of $\Theta$, including the empty set $\phi$. For example, $\Theta=\{$ actif, inactif $\}$ is the frame of discernment for a binary sensor, representing the universe of the problem, i.e., the set containing all elements (classes, states, ...). $P(\Theta)=\{\phi,\{$ actif $\},\{$ inactif $\}, \Theta\}$, is the power set, where $P(\Theta)$

has $2^{\Theta}$ elements and the element $\Theta=\{$ actif, inactif $\}$ represents uncertainty of the sensor either active or inactive. The uncertainty measure is used to represent the errors associated with the sensors because their reliability is never perfect.

Many characteristics surrounding the sensor have an impact on the quality of the sensor's observation. DST assigns a value in the interval $[0,1]$ to represent the degree of belief in the observation. This distribution of belief over the space of discernment is called evidence. A function $m: 2^{\Theta} \rightarrow[0,1]$ is then introduced and called mass function, which represents the distribution of belief and that satisfies the following two conditions

and

$$
m(\phi)=0,
$$

$$
\sum_{A \in \Theta} m(A)=1 .
$$

DST uses an interval of probabilities rather than a single probabilistic number to represent uncertainty. The lower and upper bounds of probability are called Belief and Plausibility respectively, which can be defined mass function as follows,

$$
\operatorname{Bel}(A)=\sum_{B \mid B \subseteq A} m(B),
$$

and

$$
\operatorname{Pl}(A)=\sum_{B \mid B \cap A \neq 0} m(B) .
$$

$\operatorname{Bel}(A)$ represents the degree of belief to which the evidence supports $A$. $P I(A)$ describes the degree of belief to which the evidence fails to refute $A$, that is, the degree of belief to which it remains plausible.

\section{A. Evidential operations}

The evidential operations [11-15] represent the relationships between network nodes for propagating belief distributions and are used to infer activities throughout the evidential network.

Some sensors are more sensitive to misreading or malfunctioning due to their type and where they are installed (location). The impact of evidence is discounted to reflect the sensor's credibility, in terms of discounting rate $r(0 \leq r \leq 1)$. The discounted mass function is defined as follows [11],

$$
m^{r}(A)= \begin{cases}(1-r) m(A) & A \subset \Theta \\ r+(1-r) m(\Theta) & A=\Theta\end{cases}
$$

where for $r=0$, the source is absolutely reliable; for $0 \leq r \leq 1$, the source is reliable with a rate reduction $r$ and $r=$ 1 , the source is completely unreliable.

A multivalued mapping $[11,12] \Gamma$ reflects the relationship between two frames of discernment from different sources $\left(\Theta_{A}, \Theta_{B}\right)$ representing evidence of the same problem but from different views. This multivalued mapping $\Gamma$ describes a function mapping $\Gamma: \Theta_{A} \leftarrow 2^{\Theta_{B}}$ assigning to each element $e_{i}$ of $\Theta_{A}$ a subset $\Gamma\left(e_{i}\right)$ of $\Theta_{B}$.

The evidential operation called translation [11], which relies completely on the multivalued mapping, can be used to determine the impact of evidence originally appearing on a frame of discernment on elements of a compatibly related frame of discernment. The belief distribution of the original source is then transferred to the compatible source using the multivalued mapping.

The relationship between an element $e_{i}$ of $\Theta_{A}$ and a subset $\Gamma\left(e_{i}\right)$ of $\Theta_{B}$ may be uncertain. To represent such uncertain relationship an evidential mapping [13] $\Gamma^{*}$ assigns probabilities to an element $e_{i}$ of $\Theta_{A}$ instead of a set of subsets. Belief distributions of the source $\Theta_{A}$ are propagated to the source $\Theta_{B}$ using an evidential mapping. This operation is called propagation [14]. The translation operation is a special case of propagation, in which relationships between sources $\Theta_{A}$ and $\Theta_{B}$ are certain.

When several belief distributions come from different sources on the same space of discernment $\Theta$, a new belief distribution representing the consensus of those disparate views can be produced by Dempster's rule of combination. Let $m_{1}, \ldots, m_{n o}$ mass functions on $\Theta$ representing no independent sources. $B, \ldots, C$ represent focal elements of $m_{1}, \ldots, m_{n o}$ respectively. A new mass function $m_{1, \ldots, n o}$ is formed by combining $m_{1}(\cdot) \oplus \ldots \oplus m_{n o}(\cdot): m_{1, \ldots, n o}(\cdot)$

$$
m_{1, \ldots, n o}(A)=\frac{\sum_{B \cap \ldots \cap C=A} m_{1}(B) \cdot \ldots \cdot m_{n o}(C)}{1-K},
$$

where 


$$
K=\sum_{B \cap \ldots \cap C=\phi} m_{1}(B) \cdot \ldots \cdot m_{n o}(C) .
$$

$K$ represents the measure of conflict level between the masses. The normalization factor $1-K$ can take into account these conflicts and assign any mass involved in the conflict to the null set. When $K=1$, we have a total conflict, i.e., the masses involved in the fusion process do not have an intersection in the frame of discernment. To compose a new belief distribution from dependent sources, a weighted sum operator is used.

\section{HETEROGENEOUS MULTI-SENSORS FUSION FOR FALL DETECTION}

Remote monitoring systems with alarm management (RFPAT and GARDIEN) studied in this work allows to detect distress events like person's falls, abnormal cardiac events like bradycardia. That elderly or dependent persons living alone could have. These systems constitute complementary information and their fusion can provide more reliable detection compared to systems used separately. RFPAT modality is already a fall detector and the purpose of this fusion is to secure the detection of falls difficult to identify such as soft falls or falls without impacts on the ground. Contextual information, such as the person's localization and interaction with the environment are very useful and should be interpreted as complementary information by the fusion process.

In this section, the Dempster Shafer theory is used to estimate the degrees of belief and uncertainty of potential distress events through these multiple heterogeneous sensors. An Evidential Network [5, 6] is proposed here to be introduced in the fusion process for the person status detection such as "fall" or "normal" status. Parameters from the different alarm processing modalities (such as location, motion, inclination and fall index) constitute the input evidences of the EN of interest.

\section{A. Proposed Evidential Network for fall detection}

Based on the works of Lee, Hong and Nugent [5, 6], who proposed an Evidential Network for the recognition of activities in environments such as smart homes, we propose in this work an Evidential Network for activities inference such as fall detection as shown in Figure 1. The proposed fusion approach is based on the use of the Dempster-Shafer Theory operations and rules applied to vital, actimetric and contextual information extracted from the multimodal heterogeneous alarm management system previously described in Section 2. Binary data at lower-level (IR sensors) and at higher-level (from RFPAT) constitute the input evidences of the considered network.

This network is structured as an acyclic graph. Hierarchy and links between nodes create dependencies between the different alarm management modalities (GARDIEN and RFPAT), which makes the fusion process more robust and reliable for abnormal events detections. In this network, sensors nodes are represented by circular nodes, whereas triangular nodes represent decision-making systems of various types (classifiers based on thresholds, pattern recognition algorithms or experts). Square nodes represent contextual information e.g. an object with which the person interacts or the person's location itself (e.g. in a given room). The rectangular nodes represent the activities. The connections between nodes are represented by evidential operations, as shown in the Table on the right side of Figure 1.

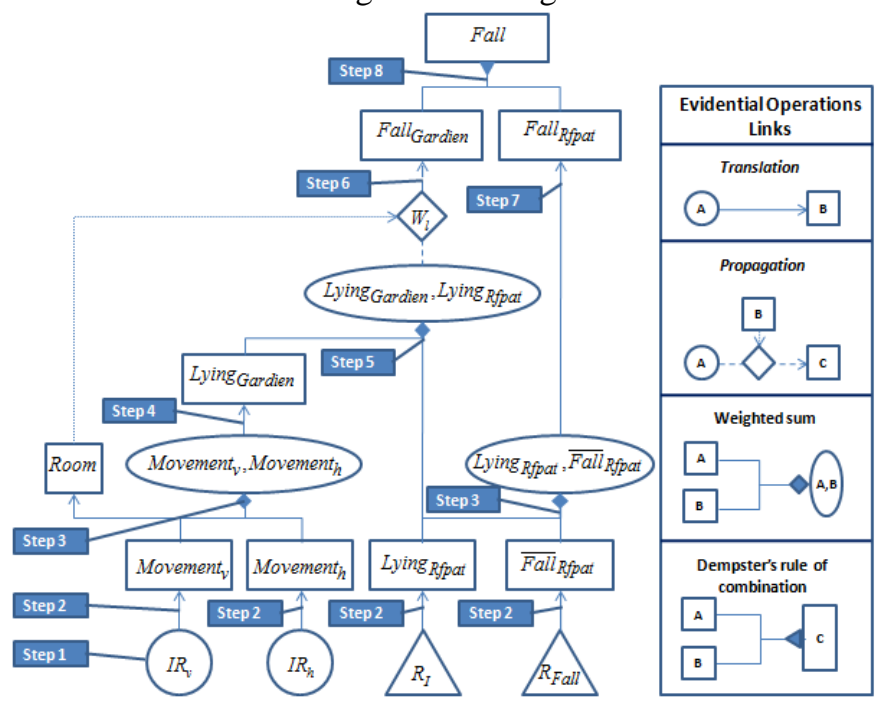

Fig. 1. Clearly the proposed network inference activities fall of person (left side). Connections between nodes represented by evidential operations (right).

\section{B. Activity inference of evidential networks}

When starting the inference process of the evidential network, we assign Belief distributions to the input nodes based on a priori sensitivity and specificity information provided by the sensors (e.g. GARDIEN) and detection devices (e.g. RFPAT) developers. Evidential operations (Section III.A) are used in the propagation process of inputs evidences towards the top nodes layer of the network providing the fall decision. Each Alarm processing modality can infer a fall belief, denoted $m(\{$ Fall $\})$, a normal belief, $m(\{\neg$ Fall $\})$, or uncertainty degree denoted $m(\{$ Fall, $\neg$ Fall $\})$. Then the overall mass functions are fused using the combination rule of Dempster-Shafer (6) to reach a consensus decision.

\section{Evidential Network inputs}

In the network of Figure 1, represented by circular nodes, the infrared sensors may have two possible states: excited (bit 1) and non-excited (bit 0). The frame of discernment of each IR sensor is $\{I R, \neg I R\}$, where $I R$ and $\neg I R$ represent a excited $\left(I R_{\text {imput }}=1\right)$ and non-excited $\left(I R_{\text {imput }}=0\right)$ sensor respectively. Nodes "IR $" R_{v}$ and "IR $R_{h}$ " represents vertical and horizontal IR sensors fields respectively placed in a given room, which is identified by the context node "room".

From the RFPAT system, triangular binary nodes " $R_{I}$ " and " $R_{\text {Fall }}$ " represents the posture parameters: "standing / sitting" or "lying down" and "fall" or "not falling", respectively. The posture activity node " $R_{I}$ " has a frame of discernment $\left\{R_{I}, \neg R_{I}\right\}$ where bits 0 and 1 represent the activities "standing / sitting" $\left(\neg R_{I}\right)$ and "lying down" $\left(R_{I}\right)$, respectively. For the 
activity node " $R_{\text {Fall }}$ ", the frame of discernment is $\left\{R_{\text {Fall }}, \neg R_{\text {Fall }}\right\}$ where bits 0 and 1 represent the activities "not falling" $\left(\neg R_{\text {Fall }}\right)$ and "fall" $\left(R_{\text {Fall }}\right)$, respectively.

\section{Evidential network inference}

The evidential inference process is summarized in eight evidential operations steps shown in Figure 1. To describe the EN inference process, we present the operations step by step based on an exemplified set of input sensors values: $I R_{v}=0$ and $I R_{h}=0$ for GARDIEN system, and $R_{I}=1$ and $R_{\text {Fall }}=1$ for RFPAT system. These values represent the states of the person: "fall", "lying down" and "immobile".

In this work, we use the node abbreviations: Movement $_{v}=$ $M_{v} ;$ Movement $_{h}=M_{h} ;$ Lying $_{\text {Rfpat }}=L_{R} ;\left(\right.$ Lying $\left._{\text {Rfpat }}, \overline{\text { Fall }}_{\text {Rfpat }}\right)=$ $\left(L_{R}, \bar{F}_{R}\right) ; \overline{\text { Fall }}_{R \text { fpat }}=\bar{F}_{R} ;\left(\right.$ Movement $_{v}$, Movement $\left._{h}\right)=$ $\left(M_{v}, M_{h}\right) ;$ Lying $_{\text {Gardien }}=L_{G} ;\left(\right.$ Lying $_{\text {Gardien }}$, Lying $\left._{R \text { fpat }}\right)=$ $\left(L_{G}, L_{R}\right) ;$ Fall $_{R f p a t}=F_{R} ;$ Fall $_{\text {Gardien }}=F_{G}$.

At the beginning of the inference we consider that each input system is $100 \%$ reliable. Hence the evidences in each input node are represented by mass functions as follows,

$$
m\left(\left\{\neg I R_{v}\right\}\right)=1 ; m\left(\left\{\neg I R_{h}\right\}\right)=1 ; m\left(\left\{R_{I}\right\}\right)=1 ; m\left(\left\{R_{\text {Fall }}\right\}\right)=1 .
$$

\section{Step 1 - Discounting sensor evidence}

Developers and manufacturers characteristics show that IR sensors (GARDIEN) work correctly with a sensitivity and specificity of $95 \%$. Thus a reduction rate of $5 \%(r=0.05)$ is assigned to each sensor. For the RFPAT modality, we assume a sensitivity of $92 \%$ and a specificity of $95 \%$, given by the evaluation experiments. So reduction rates of $8 \%(r=0.08)$ and 5\% $(r=0.05)$ are assigned to the inputs values of 1 and 0 respectively. Using (5), the discounting mass functions can be calculated as follows,

$$
\begin{aligned}
& m^{r}\left(\left\{\neg I R_{v}\right\}\right)=0,95 ; m^{r}\left(\left\{I R_{v}, \neg I R_{v}\right\}\right)=0,05 \\
& m^{r}\left(\left\{\neg I R_{h}\right\}\right)=0,95 ; m^{r}\left(\left\{I R_{h}, \neg I R_{h}\right\}\right)=0,05 \\
& m^{r}\left(\left\{R_{I}\right\}\right)=0,92 ; m^{r}\left(\left\{R_{I}, \neg R_{I}\right\}\right)=0,08 \\
& m^{r}\left(\left\{R_{\text {Fall }}\right\}\right)=0,92 ; m^{r}\left(\left\{R_{\text {Fall }}, \neg R_{\text {Fall }}\right\}\right)=0,08
\end{aligned}
$$

\section{Step 2 - Translating mass functions from sensors nodes to activities nodes}

An active IR motion sensor indicates that a person moving is interacting with the sensor. The relationships between sensor and activity nodes can be represented by a multivalued mapping. Table 1 shows the mapping of the "IR $R_{v}$ " node. We have a multivalued mapping for each input node. For reasons of lack of space, we show here only the multivalued mapping "IR $R_{v}$ " node as an example.

The mass functions on the input nodes are then translated to the associated compatible activity nodes by using the multivalued mapping.

$$
\begin{aligned}
& m\left(\left\{\neg M_{v}\right\}\right)=m^{r}\left(\left\{\neg I R_{v}\right\}\right)=0,95 ; \\
& m\left(\left\{M_{v}, \neg M_{v}\right\}\right)=m^{r}\left(\left\{I R_{v}, \neg I R_{v}\right\}\right)=0,05 ;
\end{aligned}
$$

$$
\begin{aligned}
& m\left(\left\{\neg M_{h}\right\}\right)=m^{r}\left(\left\{\neg I R_{h}\right\}\right)=0,95 ; \\
& m\left(\left\{M_{h}, \neg M_{h}\right\}\right)=m^{r}\left(\left\{I R_{h}, \neg I R_{h}\right\}\right)=0,05 ; \\
& m\left(\left\{L_{R}\right\}\right)=m^{r}\left(\left\{R_{I}\right\}\right)=0,92 ; \\
& m\left(\left\{L_{R}, \neg L_{R}\right\}\right)=m^{r}\left(\left\{R_{I}, \neg R_{I}\right\}\right)=0,08 ; \\
& m\left(\left(\bar{F}_{R}\right\}\right)=m^{r}\left(\left\{R_{\text {Fall }}\right\}\right)=0,92 ; \\
& m\left(\left(\bar{F}_{R}, \neg \bar{F}_{R}\right\}\right)=m^{r}\left(\left\{R_{\text {Fall }}, \neg R_{\text {Fall }}\right\}\right)=0,08 .
\end{aligned}
$$

TABLE I

MULTIVALUED MAPPING "IR $R_{v}$ " NODE.

\begin{tabular}{|c|l|}
\hline Relationship & Multivalued Mapping \\
\hline$I R_{v} \rightarrow M_{v}$ & $\left\{I R_{v}\right\} \rightarrow\left\{M_{v}\right\} ;\left\{\neg I R_{v}\right\} \rightarrow\left\{\neg M_{v}\right\} ;$ \\
& $\left\{I R_{v}, \neg I R_{v}\right\} \rightarrow\left\{M_{v}, \neg M_{v}\right\} ;$ \\
\hline$M v_{v} \rightarrow\left(M_{v}, M_{h}\right)$ & $\left\{M_{v}\right\} \rightarrow\left\{\left(M_{v}, M_{h}\right)\right\} ;\left\{\neg M_{v}\right\} \rightarrow\left\{\neg\left(M_{v}, M_{h}\right)\right\} ;$ \\
& $\left\{M_{v}, \neg M_{v}\right\} \rightarrow\left\{\left(M_{v}, M_{h}\right), \neg\left(M_{v}, M_{h}\right)\right\}$ \\
\hline$\left(M_{v}, M_{h}\right) \rightarrow L_{G}$ & $\left\{\left(M_{v}, M_{h}\right)\right\} \rightarrow\left\{\neg L_{G}\right\} ;\left\{\neg\left(M_{v}, M_{h}\right)\right\} \rightarrow\left\{L_{G}\right\} ;$ \\
& $\left\{\left(M_{v}, M_{h}\right), \neg\left(M_{v}, M_{h}\right)\right\} \rightarrow\left\{L_{G}, \neg L_{G}\right\}$ \\
\hline$L_{G} \rightarrow\left(L_{G}, L_{R}\right)$ & $\left\{L_{G}\right\} \rightarrow\left\{\left(L_{G}, L_{R}\right)\right\} ;\left\{\neg L_{G}\right\} \rightarrow\left\{\neg\left(L_{G}, L_{R}\right)\right\}$ \\
& $\left\{L_{G}, \neg L_{G}\right\} \rightarrow\left\{\left(L_{G}, L_{R}\right), \neg\left(L_{G}, L_{R}\right)\right\}$ \\
\hline$\left(L_{G}, L_{R}\right) \rightarrow F_{G}$ & $\left\{\left(L_{G}, L_{R}\right)\right\} \rightarrow\left\{F_{G}\right\} ;\left\{\neg\left(L_{G}, L_{R}\right)\right\} \rightarrow\left\{\neg F_{G}\right\} ;$ \\
& $\left\{\left(L_{G}, L_{R}\right), \neg\left(L_{G}, L_{R}\right)\right\} \rightarrow\left\{F_{G}, \neg F_{G}\right\} ;$ \\
\hline
\end{tabular}

Step 3 - Composite activity node using a weighted sum operator

The "($\left.M_{v}, M_{h}\right)$ " composite activity node is composed of " $M_{v}$ " and " $M_{h}$ " activity nodes. This node represents the IR sensors based-activity nodes combination to determine the "lying down" posture. In the same way, the "( $\left.L_{R}, \bar{F}_{R}\right)$ " composite activity node is composed of " $L_{R}$ " and " $\bar{F}_{R}$ " activity nodes. This node represents the combination between the posture and fall parameters to reinforce the fall belief of the RFPAT system. The " $M_{v}$ " and " $M_{h}$ " nodes mass functions are, at first, translated using the multivalued mapping to " $\left(M_{v}, M_{h}\right)_{v}$ " and " $\left(M_{v}, M_{h}\right)_{h}$ " intermediary composite activity nodes, respectively. The same procedure is used on the " $\left(L_{R}, \bar{F}_{R}\right)_{L}$ " and " $\left(L_{R}, \bar{F}_{R}\right)_{F}$ " intermediary composite activity nodes as follows.

$$
\begin{aligned}
& m\left(\left\{\neg\left(M_{v}, M_{h}\right)\right\}\right)_{v}=m\left(\left\{\neg M_{v}\right\}\right)=0,95 \\
& m\left(\left\{\left(M_{v}, M_{h}\right), \neg\left(M_{v}, M_{h}\right)\right\}\right)_{v}=m\left(\left\{M_{v}, \neg M_{v}\right\}\right)=0,05 \\
& m\left(\left\{\neg\left(M_{v}, M_{h}\right)\right\}\right)_{h}=m\left(\left\{\neg M_{h}\right\}\right)=0,95 \\
& m\left(\left\{\left(M_{v}, M_{h}\right), \neg\left(M_{v}, M_{h}\right)\right\}\right)_{h}=m\left(\left\{M_{h}, \neg M_{h}\right\}\right)=0,05 \\
& m\left(\left\{L_{R}, \bar{F}_{R}\right\}\right)_{L}=m\left(\left\{L_{R}\right\}\right)=0,92 \\
& m\left(\left\{\left(L_{R}, \bar{F}_{R}\right), \neg\left(L_{R}, \bar{F}_{R}\right)\right\}\right)_{L}=m\left(\left\{L_{R}, \neg L_{R}\right\}\right)=0,08 \\
& m\left(\left(\left\{L_{R}, \bar{F}_{R}\right\}\right)_{F}=m\left(\left(\bar{F}_{R}\right\}\right)=0,92\right. \\
& m\left(\left(\left\{L_{R}, \bar{F}_{R}\right), \neg\left(L_{R}, \bar{F}_{R}\right)\right\}\right)_{F}=m\left(\left(\bar{F}_{R}, \neg \bar{F}_{R}\right\}\right)=0,08
\end{aligned}
$$

The "( $\left.M_{v}, M_{h}\right)$ " composite activity node is the result of a weighted sum of the " $\left(M_{v}, M_{h}\right)_{v}$ " and " $\left(M_{v}, M_{h}\right)_{h}$ " nodes and, in the same way, the " $\left(L_{R}, \bar{F}_{R}\right)$ " composite activity node is the result of a weighted sum of the " $\left(L_{R}, \bar{F}_{R}\right)_{L}$ " and " $\left(L_{R}, \bar{F}_{R}\right)_{F}$ " 
nodes. In the weighted sum, the weight choice depends on the reliability of the nodes. The weights, empirically chosen, are: $w_{v}=0,2, w_{h}=0,8, w_{L}=0,4, w_{F}=0,6$. Then we have:

$$
\begin{aligned}
& m\left(\left\{\neg\left(M_{v}, M_{h}\right)\right\}\right)=w_{v} m\left(\left\{\neg\left(M_{v}, M_{h}\right)\right\}\right)_{v}+w_{h} m\left(\left\{\neg\left(M_{v}, M_{h}\right)\right\}\right) h \\
& =0,2 \cdot 0,95+0,8 \cdot 0,95=0,95 \\
& m\left(\left\{\left(M_{v}, M_{h}\right), \neg\left(M_{v}, M_{h}\right)\right\}\right)=w_{v} m\left(\left\{\left(M_{v}, M_{h}\right), \neg\left(M_{v}, M_{h}\right)\right\}\right)_{v}+ \\
& w_{h} m\left(\left\{\left(M_{v}, M_{h}\right), \neg\left(M_{v}, M_{h}\right)\right\}\right)_{h}=0,2 \cdot 0,05+0,8 \cdot 0,05=0,05 \\
& m\left(\left\{L_{R}, \bar{F}_{R}\right\}\right)=w_{L} m\left(\left\{L_{R}, \bar{F}_{R}\right\}\right)_{L}+w_{F} m\left(\left\{L_{R}, \bar{F}_{R}\right\}\right)_{F} \\
& =0,4 \cdot 0,92+0,6 \cdot 0,92=0,92 \\
& m\left(\left\{\left(L_{R}, \bar{F}_{R}\right),-\left(L_{R}, \bar{F}_{R}\right)\right\}\right)=w_{L} m\left(\left\{\left(L_{R}, \bar{F}_{R}\right), \neg\left(L_{R}, \bar{F}_{R}\right)\right\}\right)_{L} \\
& +w_{F} m\left(\left(\left(L_{R}, \bar{F}_{R}\right), \neg\left(L_{R}, \bar{F}_{R}\right)\right\}\right)_{F}=0,4 \cdot 0,08+0,6 \cdot 0,08=0,08
\end{aligned}
$$

Step 4 - Translating mass functions from the composite activity nodes to the activities nodes

The " $\left(M_{v}, M_{h}\right)$ " node represents the "lying down" state of person, then the belief distribution in this node is translated to the " $L_{G}$ " node (lying down node). This process occurs in a reversed mode, which is due to the fact that the "lying down" activity is inferred from the non-excitation of IR sensors.

$m\left(\left\{L_{G}\right\}\right)=m\left(\left\{\neg\left(M_{v}, M_{h}\right)\right\}\right)=0,95$

$m\left(\left\{L_{G}, \neg L_{G}\right\}\right)=m\left(\left\{\left(M_{v}, M_{h}\right), \neg\left(M_{v}, M_{h}\right)\right\}\right)=0,05$

\section{Step 5 - Composite activity node using a weighted sum} operator

The " $\left(L_{G}, L_{R}\right)$ " composite activity node is formed by the " $L_{G}$ " (GARDIEN) and " $L_{R}$ " (RFPAT) activity nodes. The mass functions in " $L_{G}$ " and " $L_{R}$ " are then translated to " $\left(L_{G}, L_{R}\right)_{G}$ " and " $\left(L_{G}, L_{R}\right)_{R}$ " nodes, respectively :

$$
\begin{aligned}
& m\left(\left\{L_{G}, L_{R}\right\}\right)_{G}=m\left(\left\{L_{G}\right\}\right)=0,95 \\
& m\left(\left\{\left(L_{G}, L_{R}\right), \neg\left(L_{G}, L_{R}\right)\right\}\right)_{G}=m\left(\left\{L_{G}, \neg L_{G}\right\}\right)=0,05 \\
& m\left(\left\{L_{G}, L_{R}\right\}\right)_{R}=m\left(\left\{L_{R}\right\}\right)=0,92 \\
& m\left(\left\{\left(L_{G}, L_{R}\right), \neg\left(L_{G}, L_{R}\right)\right\}\right)_{R}=m\left(\left\{L_{R}, \neg L_{R}\right\}\right)=0,08
\end{aligned}
$$

The weights, chosen experimentally, are: $w_{L g}=0,2$ (GARDIEN posture) and $w_{L r}=0,8$ (RFPAT posture). This choice reflects a larger credit to the RFPAT posture sensor due to its reliability, because it features sensors based on accelerometers with high inclination measurement precision. The weighted sum :

$$
\begin{array}{rl}
m\left(\left\{L_{G}, L_{R}\right\}\right) & =w_{L g} m\left(\left\{A L_{G}, A L_{R}\right\}\right)_{G}+w_{L r} m\left(\left\{A L_{G}, A L_{R}\right\}\right)_{R} \\
& =0,2 \cdot 0,95+0,8 \cdot 0,92=0,926 \\
m\left(\left\{\left(L_{G}, L_{R}\right),\right.\right. & \left.\left.\neg\left(L_{G}, L_{R}\right)\right\}\right)=w_{L g} m\left(\left\{\left(L_{G}, L_{R}\right), \neg\left(L_{G}, L_{R}\right)\right\}\right)_{G}+ \\
\quad+w_{L r} & m\left(\left\{\left(L_{G}, L_{R}\right), \neg\left(L_{G}, L_{R}\right)\right\}\right)_{R}=0,2 \cdot 0,05+0,8 \cdot 0,05=0,074
\end{array}
$$

Step 6 - Inferring from a activity node to a deduced activity node : pre-fused GARDIEN data obtaining

The "fall" activity is made ambiguous by the "lying down" activity of the person, with these two activities being uncertain and defined heuristically. The mass function in the "lying down" activity node is propagated in the "fall" activity node by the evidential mapping. For this inference, the person is in the kitchen, so we have the following relationship given by the evidential mapping

$$
\begin{aligned}
& m\left(\left\{L_{G}, L_{R}\right\} \stackrel{\text { Kitchen }}{\longrightarrow}\left\{F_{G}\right\}\right)=0,9 \\
& m\left(\left\{L_{G}, L_{R}\right\} \stackrel{\text { Kitchen }}{\longrightarrow}\left\{F_{G}, \neg F_{G}\right\}\right)=0,1 \\
& m\left(\left\{\neg\left(L_{G}, L_{R}\right)\right\} \stackrel{\text { Kitchen }}{\longrightarrow}\left\{\neg F_{G}\right\}\right)=1 \\
& m\left(\left\{L_{G}, \neg L_{R}\right\} \stackrel{\text { Kitchen }}{\longrightarrow}\left\{F_{G}, \neg F_{G}\right\}\right)=1
\end{aligned}
$$

Using the belief distribution on the "lying down" activity node is (Step 5), the inference of "Fall" activity node result is:

$$
\begin{aligned}
& m\left(\left\{F_{G}\right\}\right)=m\left(\left\{L_{G}, L_{R}\right\}\right) \cdot m\left(\left\{L_{G}, L_{R}\right\} \stackrel{\text { kitchen }}{\longrightarrow}\left\{F_{G}\right\}\right) \\
& =0,926 \cdot 0,9=0,8334 \\
& m\left(\left\{F_{G}, \neg F_{G}\right\}\right)=m\left(\left\{L_{G}, L_{R}\right\}\right) \cdot m\left(\left\{L_{G}, L_{R}\right\} \stackrel{\text { kitchen }}{\longrightarrow}\left\{F_{G}, \neg F_{G}\right\}\right)+ \\
& m\left(\left\{\left(L_{G}, L_{R}\right), \neg\left(L_{G}, L_{R}\right)\right\}\right) \cdot m\left(\left\{\left(L_{G}, L_{R}\right), \neg\left(L_{G}, L_{R}\right)\right\} \stackrel{\text { kitchen }}{\longrightarrow}\left\{F_{G}, \neg F_{G}\right\}\right) \\
& =0,926 \cdot 0,1+0,074 \cdot 1=0,1666
\end{aligned}
$$

The result of this inference for the GARDIEN system is a "fall" belief of $m\left(\left\{F_{G}\right\}\right)=0,8334$ and a "fall" uncertainty of $m\left(\left\{F_{G}, \neg F_{G}\right\}\right)=0,1666$.

Step 7 - Translating mass functions from the composite activity node to the activity node : pre-fused RFPAT data obtaining

The belief distribution of " $\left(L_{R}, \bar{F}_{R}\right)$ " composite activity node is translated to the " $F_{R}$ " activity node. The translated mass, given by the multivalued mapping, then becomes,

$m\left(\left\{F_{R}\right\}\right)=m\left(\left\{L_{R}, \bar{F}_{R}\right\}\right)=0,92$
$m\left(\left\{F_{R}, \neg F_{R}\right\}\right)=m\left(\left\{\left(L_{R}, \bar{F}_{R}\right), \neg\left(L_{R}, \bar{F}_{R}\right)\right\}\right)=0,08$

Step 8 - Combining mass function on an activity node - final fused GARDIEN and RFPAT data obtention

In this step, each system (RFPAT and GARDIEN) has a belief distribution for the fall activity. The combination rule of Dempster Shafer (6) can reach a new belief distribution that represents the consensus of the distributions of the two belief systems. The belief distribution of the GARDIEN system inferred on Step 6 is: $m\left(\left\{F_{G}\right\}\right)=0,8334$ and $m\left(\left\{F_{G}, \neg F_{G}\right\}\right)=0,1666$. The belief distribution of the RFPAT system inferred on Step 7 is: $m\left(\left\{F_{R}\right\}\right)=0,92$ and $m\left(\left\{F_{R}, \neg F_{R}\right\}\right)=0,08$. Using the combination rule of Dempster Shafer we finally have:

$$
\begin{gathered}
m_{G, R}(\text { Fall })=\frac{0,8334 \cdot 0,92+0,8334 \cdot 0,08+0,92 \cdot 0,1666}{1-0}=0,986672 \\
m_{G, R}(\text { Fall }, \neg \text { Fall })=\frac{0,08 \cdot 0,1666}{1-0}=0,013328
\end{gathered}
$$

where $K=0$. The result is a fall belief of $m_{G, R}($ Fall $)=0,986672$ and a fall uncertainty of $m_{G, R}($ Fall, $\neg$ Fall $)=0,013328$, for $K=0$, because we have no null intersection, so no conflict between the two modalities were considered. The DS rule of combination can increase the 
recognition of fall belief. It represents the result of the belief consensus of the fusioned systems (GARDIEN and RFPAT).

\section{PRELIMINARY RESEARCH RESUlTS}

The evaluation of the proposed method was performed on data recorded at Telecom SudParis. These databases consist of simulated scenarios with normal and falls situations. In these databases we have several scenarios of classical fall (rather violent) and soft falls (with low acceleration). These databases contain 33 fall and 5 normal scenarios. Among the 33 fall scenarios, we have 17 soft falls and 16 hard falls. The purpose of our evidential network based fusion is to detect in very specific case of soft falls.

These databases are then used to evaluate the proposed evidential network. To evaluate the evidential network performance a confusion matrix has been computed on normal and fall situations, as shown in Table 2.

TABLE 2

CONFUSION MATRIX OF THE EN FUSION.

\begin{tabular}{|l|l|l|l|}
\hline \multicolumn{2}{|c|}{ ConfuSION MATRIX OF THE EN FUSION. } \\
\cline { 3 - 4 } & \multicolumn{2}{c|}{ EN fusion } \\
\cline { 3 - 4 } Confusion matrix & Normal & Fall \\
\hline \multirow{2}{*}{ Scenarios } & Normal & 5 & 0 \\
\cline { 2 - 4 } & Fall & 2 & 31 \\
\hline
\end{tabular}

In Table 2, "Fall" is a fall detected event and "Normal" is when no fall is detected. The EN fusion has not detected only 2 fall cases which the network is not adapted to: the first case is a soft fall on a sofa; the second case is a soft fall in a bedroom. Concerning the fall on sofa, the system is not yet adapted: the person falls without impact and ends in a sitting posture / standing. This network needs the lying down posture to detect the fall. Concerning the case of soft fall into in a bedroom the EN fusion has permitted to detect this difficult situation for the separated modalities. In this case the context of location "room" is very uncertain and it has a low weight heuristic because the posture "lying down" in the room does not necessarily mean that the person has fallen. It may be a situation where the person sleeps. This problem can be resolved with the introduction of other modalities (video, for example) in the network where we can have more positional accuracy to distinguish for example if the person is lying because she is in bed or it's down. The EN fusion presented promising good performance (sensitive of 93,94\%), in particular for soft fall cases, compared to separated modalities. The values of alone RFPAT system performance are not communicated for reasons of confidentiality due to a patent process underway. Furthermore we need to evaluate our system on a more extended database to confirm the contribution of EN fusion to the difficult falls to detect.

\section{CONCLUSION AND PERSPECTIVES}

Evidential Network fusion of GARDIEN and RFPAT télévigilance modalities improved the global fall detection sensitivity with regard to separate use of the two modalities: indeed the first conducted experiments have shown good fall detection performance, which is a very promising for the future use of such evidential network-based fusion and it also demonstrates the interest to perform a multimodal fusion. Moreover such an evidential network is modular and can detect falls even if the RFPAT system is not present in the fusion process. This network is still under development and extension. For future work we propose an extension of EN to the inference of various distress situations by adding more contextual information and parameters such as pulse rate, activity, abnormal sounds, in order to better represent the occurring distress situations. We also propose a comparison with other decision methods such as Bayesian Networks and Logic Fuzzy.

\section{ACKNOWLEDGMENT}

This research is funded by the FP7 European Project ISTCompanionAble (www.companionable.net).

\section{REFERENCES}

[1] Becker, A. and Naïm, P. (1999). Les réseaux bayésiens. Eyrolles, eyrolles edition.

[2] B. Ben Yaghlane, Ph. Smets, K. Mellouli, Directed evidential networks with conditional belief functions. In: Nielsen TD, Zhang NL (eds.) Proceedings of ECSQARU, 2003

[3] A. Benavoli, B. Ristic, A. Farina, M. Oxenham, L. Chisci "An approach to Threat Assessment Based on Evidential Networks", Proc. Int. Conf. Fusion 2007 Conference, Quebec City, Canada, July 9-12, 2007.

[4] Simon C., Weber P.: Evidential networks for reliability analysis and performance evaluation of systems with imprecise knowledge. IEEE Trans on Reliability, 2009.

[5] Hyun Lee; Jae Sung Choi; Elmasri, R.; , "Sensor Data Fusion Using DSm Theory for Activity Recognition under Uncertainty in HomeBased Care," Advanced Information Networking and Applications, 2009. AINA '09. International Conference on, May 2009.

[6] Hong, X., Nugent, C., Mulvenna, M., McClean, S., Scotney, B., Devlin, S.: Evidential fusion of sensor data for activity recognition in smart homes, Pervasive and Mobile Computing, Volume 5, Issue 3, Pervasive Health and Wellness Management, June 2009.

[7] H. Medjahed, D. Istrate, J. Boudy and B. Dorizzi.: A Fuzzy Logic System for Home Elderly People Monitoring (EMUTEM). In: Fuzzy Systems 2009, Prague, Czech Republic.

[8] Steenkeste , F., Bocquet, H., Chan, M., Vellas, B.: Remote monitoring system for elders in a geriatric hospital., international conference on aging, Arlington December 1999.

[9] J.L. Baldinger et al.: Tele-surveillance system for patient at home. The MEDEVILLE system. ICCHP, Paris July 2004.

[10] Istrate, D., Castelli, E., Vacher, M., Besacier, L., Serignat, J. F.: Information Extraction from Sound for Medical Telemonitoring”, IEEE Transactions april 2006.

[11] J.D. Lowrance, T.D. Garvey, T.M. Strat, A framework for evidentialreasoning systems, in: Proc. of 5th National Conf. of the American Assoc. for Artifitial Intelligence, 1986.

[12] Strat, T.M.: The generation of explanations within evidential reasoning, in: Proceedings of IJCAI, 1987, pp. 1097_1104.

[13] W. Liu, J. Hong, M.F. McTear, J.G. Hughes, An extended framework for evidential reasoining systems, International Journal of Pattern Recognition and AI (1993) 441_457.

[14] X. Hong, Heuristic Knowledge Representation and Evidence Combination Parallelization, Ph.D. thesis, University of Ulster, 2001.

[15] Paulo A. Cavalcante A. et al.: Fusion multi-capteurs hétérogène basée sur un Réseau d'Evidence pour la détection de chute. In : ASSISTH conf. 2011. 